LETTER TO THE EDITOR

\title{
Integrating Tobacco Control Measures with COVID-19 Control Programs: A Multisectoral Model
}

\author{
Shivashankar Kengadaran ${ }^{1}$, Anusha Divvi², Sivabalakumaran Kengadaran ${ }^{3}$
}

\begin{abstract}
The first case of COVID-19 was reported in Wuhan, China, in December 2019. From then, there was a rapid spread of disease across countries resulting in a heavy toll of life. Most of the countries responded to the situation by immediate lockdown. Healthcare services were completely revamped and only emergency services were provided other than COVID-19 services. In India, more than one lakh cases were diagnosed; of which 3,000 were reported dead. Although the numbers show an increase in COVID-19 cases, the mortality of COVID-19 is not comparable to the tobacco epidemic in India. The lockdown has provided tobacco users with changed social circumstances and self-isolation, which makes quitting easier. However, following the lift of lockdown there is a peak expected in both COVID-19 cases and tobacco use. It is wiser to captivate on the existing scenario and integrate COVID-19 and tobacco control measures so that both the conditions can be handled effectively.

SBV Journal of Basic, Clinical and Applied Health Science (2020): 10.5005/jp-journals-10082-02249
\end{abstract}

An epidemic outbreak of novel coronavirus was first reported in Wuhan, China, in December 2019. From then, there was a rapid spread of the disease across countries resulting in a heavy toll of life. ${ }^{1}$ Most of the countries responded to the situation by immediate international and interstate lockdown. Healthcare services were completely revamped and only emergency medical and dental services were provided other than COVID-19 services. All the available manpower was utilized for combating the spread of this deadly disease. As of 25 May 2020, more than one lakh active COVID-19 cases were diagnosed; of which around 3,000 were reported dead in India. ${ }^{2}$ Although the numbers show an increase in COVID-19 cases, the mortality of COVID-19 is not comparable to the tobacco epidemic in India. The prevalence of tobacco use was highest in the Southeast Asia region with India as the second largest consumer of tobacco. According to the WHO, India is home for $12 \%$ of the world's smokers and more than 10 million die each year due to tobacco in India. ${ }^{3}$ This outweighs the destruction caused by COVID-19 that could never match the relentless and growing annual toll from tobacco use. ${ }^{4}$ Studies also suggested that smokers having COVID-19 had severe symptoms than nonsmokers. ${ }^{5}$

Tobacco products manufacture was suspended, across all countries, as an effect of the lockdown. Despite this, the sale of tobacco showed an upward trend in most of the countries. However, unlike other countries, there was a restricted tobacco trade and availability in India. Researches show that changed social circumstances and self-isolation make quitting easier. ${ }^{4,6}$ The lockdown has provided the users with both the above-mentioned environment to facilitate quitting. However, the lockdown is a temporary process for management of COVID-19 and has been eased from the mid of May and will be completely lifted from the end of the month. The lift of lockdown with changed circumstances imposing economic burden, joblessness, and stress both physical and mental imposed due to COVID-19 and increased availability of tobacco products may act as triggers leading to relapse of the habit.

Integrating new programs in the preexisting program can be more effective than planning and implementing parallel programs. Tobacco control measures can be integrated into COVID-19 control programs. We recommend extending COVID-19 telecounseling services to tobacco users; listing anti-tobacco counseling under emergency care and effective functioning of all anti-tobacco \begin{tabular}{l}
\hline 1,2Department of Public Health Dentistry, Indira Gandhi Institute of \\
Dental Sciences, Puducherry, India \\
${ }^{3}$ Department of Anesthesia, Government Medical College, Chengalpattu,
\end{tabular} 3 Department of Anes
Tamil Nadu, India

Corresponding Author: Shivashankar Kengadaran, Department of Public Health Dentistry, Indira Gandhi Institute of Dental Sciences, Puducherry, India, Phone: +91 9003949330, e-mail: shiva.freee@gmail.com

How to cite this article: Kengadaran S, Divvi A, Kengadaran S. Integrating Tobacco Control Measures with COVID-19 Control Programs: A Multisectoral Model. J Basic Clin Appl Health Sci 2020; 3(3):133.

Source of support: Nil

Conflict of interest: None

cells both in medical and dental institutions; including nicotine gums, patches, bupropion, and varenicline along with HCQs to the essential drug list and ensuring adequate availability of these drugs; and including health education messages and warnings like smoking and secondhand smoking lead to severe COVID-19 symptoms along with the golden rules to follow during COVID-19.

\section{References}

1. WHO, Coronavirus Disease (COVID-19) Outbreak: Rights, Roles, and Responsibilities of Health Workers, Including Key Considerations for Occupational Safety and Health. Available at: https://www.who. int/docs/default-source/coronaviruse/who-rights-roles-respon-hwcovid-19.pdf?sfvrsn=bcabd401_0 Last accessed: 24 April 2020.

2. Ministry of Health and Family Welfare, GOI. Available at: www.mohfw. gov.in. Last accessed: 24 April 2020.

3. Reddy KS, Gupta PC. Tobacco control in India. New Delhi: Ministry of health and family welfare, Government of India; 2004. pp. 43-47.

4. Hefler M, Gartner CE. The tobacco industry in the time of COVID-19: time to shut it down? Tob Control 2020;29(3):245-246. DOI: 10.1136/ tobaccocontrol-2020-055807.

5. Vardavas $\mathrm{Cl}$, Nikitara K. COVID-19, and smoking: a systematic review of the evidence. Tob Induc Dis 2020;18(March):20. DOI: 10.18332/tid/119324.

6. Chaiton MO, Mecredy G, Cohen J. Tobacco retail availability and risk of relapse among smokers who make a quit attempt: a populationbased cohort study. Tob Control 2018;27(2):163-169. DOI: 10.1136/ tobaccocontrol-2016-053490. 